\title{
Free Vascularized Fibular Graft for Distal Tibial and Ankle Arthrodesis
}

\author{
Adam B. Wallace, BS ${ }^{1}$ Sean M. Devitt, MD ${ }^{1}$ Tyler M. Kreitz, MD ${ }^{2}$ Steven M. Raikin, MD \\ Patrick Joseph Greaney, Jr., MD ${ }^{1}$ \\ ${ }^{1}$ Division of Plastic Surgery, Thomas Jefferson University Hospital, \\ Philadelphia, Pennsylvania \\ 2 Department of Orthopedic Surgery, Thomas Jefferson University \\ Hospitals, Philadelphia, Pennsylvania \\ Address for correspondence Patrick Joseph Greaney, Jr., MD, Division \\ of Plastic Surgery, Thomas Jefferson University Hospital, 840 Walnut \\ Street 15th Floor, Philadelphia, PA 19107 \\ (e-mail: greaney_jmc2001@yahoo.com).
}

J Reconstr Microsurg Open 2016;1:111-116.

\begin{abstract}
Background The use of free vascularized fibular graft (FVFG) for proximal and midshaft tibial reconstruction is well documented in the literature. However, literature documenting distal tibial and proximal ankle reconstruction using this technique is lacking. The purpose of this case report is to demonstrate the osteocutaneous fibular free flap as a viable limb-sparing option to patients who previously required amputation in similar circumstances.

Methods The patient is a 39-year-old man who sustained a traumatic distal tibial pilon fracture. He underwent open reduction and internal fixation, which was complicated by osteomyelitis requiring multiple debridements and ultimately, resection of necrotic tissue. The resulting distal tibial defect measured $12 \mathrm{~cm}$, including the talus. In an attempt to salvage the extremity, an FVFG was performed using the contralateral fibula. The harvested fibula was inserted proximally into the intramedullary canal of the tibia and impacted distally into the talar dome.

Results To date, the patient's postoperative course was notable for minor wound healing issues which resolved. Postoperative computed tomography confirmed fusion, allowing for weight-bearing and removal of the external fixator.

\section{Keywords}

- free vascularized fibular graft

- ankle reconstruction

- limb salvage

Conclusion Reconstruction of distal tibial defects with ankle involvement is a challenging operation for orthopedic and plastic surgeons. We describe a case in which a $12 \mathrm{~cm}$ tibial defect in conjunction with a talar defect was successfully reconstructed with a free vascularized fibular graft. We believe that this is a safe and viable option for those wishing to avoid amputation.
\end{abstract}

The free vascularized fibular graft (FVFG) to the tibia was first described by Taylor et al in $1975 .{ }^{1}$ Since then, the operation has evolved to include transfer of adjacent muscle, and in 1983 Chen and Yan described an osteocutaneous flap. ${ }^{2}$ These advancements have provided a limb-sparing option to those patients once destined for amputation. Indications for these procedures now include trauma, chronic osteomyelitis, wide resection of malignant tumor, and pseudoarthrosis of long bones. ${ }^{3}$

received

April 21, 2016

accepted

May 26, 2016

published online

July 11, 2016
Prior to the advent of these procedures, complex fractures of the tibia were treated with autologous, nonvascularized cancellous bone grafting. Nonunion and complication rates were high, often leading to delayed amputation. Nonvascularized fibular autografts also showed high complication rates and particularly high rates of nonunion and stress fractures. Attempts to reduce these complications led to the proposal for vascularized grafts. Free vascularized flaps have quicker

Copyright $\odot 2016$ by Thieme Medical Publishers, Inc., 333 Seventh Avenue, New York, NY 10001, USA. Tel: +1(212) 584-4662.
License terms

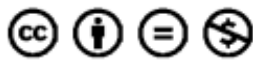

DOI http://dx.doi.org/ 10.1055/s-0036-1584937. ISSN 2377-0813. 
healing time, faster functional recovery and fewer complication rates, making them the treatment of choice for large tibial defects. $^{2}$

Due to high complication rates from nonvascularized bone grafts to large defects, tibial defects greater than $6 \mathrm{~cm}$ generally require microvascular bone grafts. The fibula has many advantages for use as a donor vascularized bone graft to the tibia. It is a compact bone, making it suitable for weightbearing and can provide a graft up to $20 \mathrm{~cm}$ of length, if desired. Additionally, the peroneal vessels are relatively large and easy to anastamose. ${ }^{4}$ Furthermore, the pedicle runs the length of the fibula so the entire graft can be taken with one pedicle. This holds true for the fibula alone, and for an osteocutaneous or osteomusculocutaneous graft. The blood supply to the skin and adjacent muscle comes from the musculocutaneous branches of the peroneal artery. The vascular anatomy, pedicle length, and bone length provide the graft with great versatility. When tibial defects are small, the harvested fibula can be folded on itself in the so-called "double-barrel" technique, preserving the vascular pedicle, and adding structural support. Surgeons have the option of harvesting both fibulas to cover a single defect. ${ }^{5}$

There are multiple methods of harvest and inset that can be utilized when reconstructing the lower extremity with a vascularized fibular graft. Beris et al describe the common approaches. ${ }^{6}$ If longitudinal growth is still desired in a child, the proximal fibula with epiphysis can be harvested. An anterior approach is taken with the anterior tibial artery and branches of the pedicle. This flap has been successful for both proximal femur, as well as humeral and distal radial reconstructions. ${ }^{7,8}$ When the defect only includes the cortex, the fibular graft can be used as an onlay graft. This method has been utilized in instances of radiation induced long-bone fractures. With this technique, the fibula is osteotomized along its longitudinal axis with the length being dependent on the defect.

In 1993, Capanna et al described the hybrid graft, combining a bone allograft in addition to the vascularized fibula graft. This hybrid graft proved to be more structurally competent and has demonstrated excellent results following massive bone resection often required with malignancy of the lower extremity. This technique takes a lateral approach and uses the peroneal artery as the pedicle. This peroneal pedicle provides both endosteal and periosteal branches, maintaining flow to the medullary canal as well as the cortex. ${ }^{9}$ Unlike the earlier described proximal fibular graft containing the epiphysis, this graft spares at least $6 \mathrm{~cm}$ both proximally and distally to maintain knee and ankle stability of the donor leg. After medullary reaming of the bony allograft, the donor fibula graft is placed in the allograft intramedullary canal. This hybrid graft can now be used to bridge the long-bone defect. ${ }^{10}$ Excellent results have been seen with diaphyseal defects of the tibia and femur. However, this technique may not be applicable to distal tibial and ankle defects.

Traditionally, the FVFG has been reserved for head and neck reconstructions. More recently, with the advent of the previously described techniques, midshaft tibia and femur defects can now be reconstructed. However, complex frac- tures of the tibio-talar joint with bone and soft tissue defects cannot be reconstructed using conventional methods. For this reason, surgeons have not opted for a vascular tissue transfer to the ankle joint in cases of trauma or osteomyelitis.

The use of FVFG for proximal and midshaft tibial reconstruction is well documented in the literature. There are multiple techniques available, each with their own indications. In this case, we describe a novel modified technique used for distal tibia and ankle arthrodesis. To our knowledge, there are no reports of distal tibial defect bridging combined with ankle arthrodesis using this technique. The purpose of this case report is to demonstrate the osteocutaneous fibular free flap as a viable option for these patients as well.

\section{Case Presentation}

\section{Patient Background and Initial Wound Management}

The patient was a 39-year-old otherwise healthy man who sustained a right distal tibial pilon fracture after a fall from a balcony. He was treated at an outside institution with open reduction and internal fixation. His postoperative course was complicated by hardware infection and wound breakdown requiring several debridements and courses of antibiotics. The patient presented to our practice for further management of his wound.

Upon examining the infected wound with exposed hardware, the patient was admitted to the hospital, started on broad-spectrum antibiotics, and seen by the orthopedic service. Plain films of the ankle ( - Fig. 1 ) revealed nonunion of the distal tibia. The patient was taken to the operating

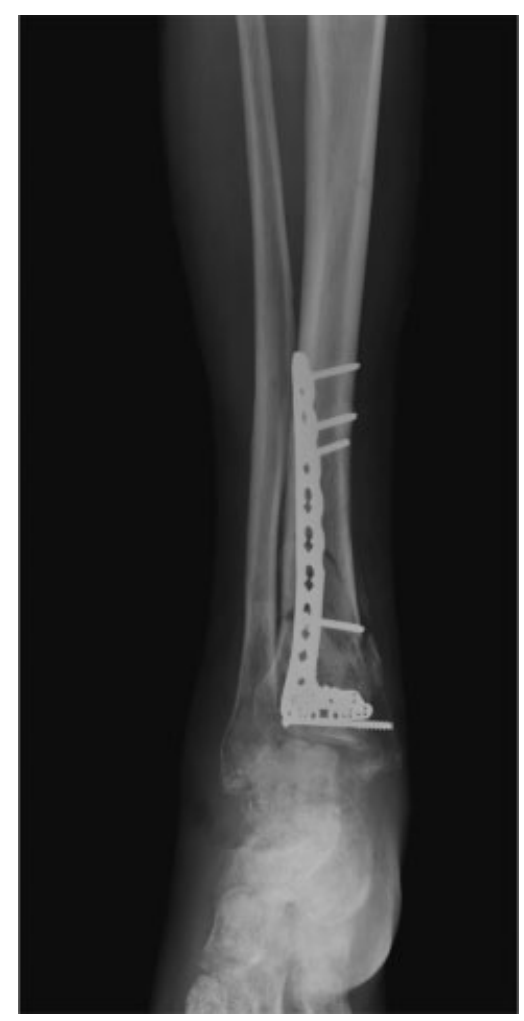

Fig. 1 Preop nonunion of tibial fracture. 


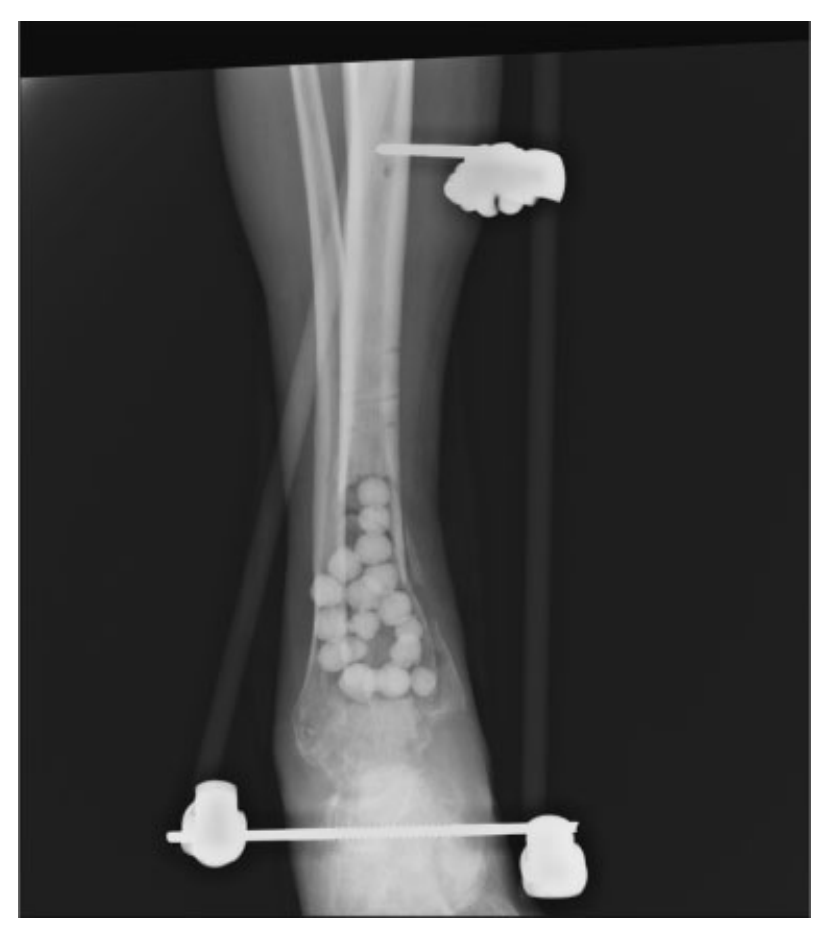

Fig. 2 Post debridement of necrotic bone and placement of antibiotic beads.

room in conjunction with the orthopedic service for hardware removal and staged debridements. The metaphyseal area of the distal tibia was found to be largely necrotic, leaving a $12 \mathrm{~cm}$ segmental defect in the distal tibia including the talus. The patient was treated for 3 months with antibiotic impregnated beads and an external fixator (-Fig. 2).

\section{Preoperative Discussion and Imaging}

Given the extent of resection of the distal tibia and ankle, the patient was offered two treatment options from the multidisciplinary team consisting of plastic surgery and foot and ankle teams. He was offered a below-knee amputation with prosthetic limb fitting or a vascularized bone graft reconstruction for limb salvage. After 3 months of antibiotic bead treatment in an external fixator, the patient opted for reconstruction with an osteocutaneous FVFG. Preoperative computed tomography angiography showed patent 3 vessel runoff in both legs.

\section{Surgical Technique}

The contralateral leg was chosen for the harvest site of the fibular free flap. The donor leg landmarks were identified (-Fig. 3) including identification of soleal and septal perforators. The dissection was carried down to the lateral septum where a single proximal septal perforator was present and a soleal perforator was spared to optimize blood supply to the skin island. The fibula was marked for osteotomy approximately $6 \mathrm{~cm}$ from the ankle joint and a similar distance from the knee to preserve joint stability. The bone was osteotomized using an oscillating saw, and the osteocutaneous free flap was isolated with preservation of the peroneal pedicle (-Fig. 4).

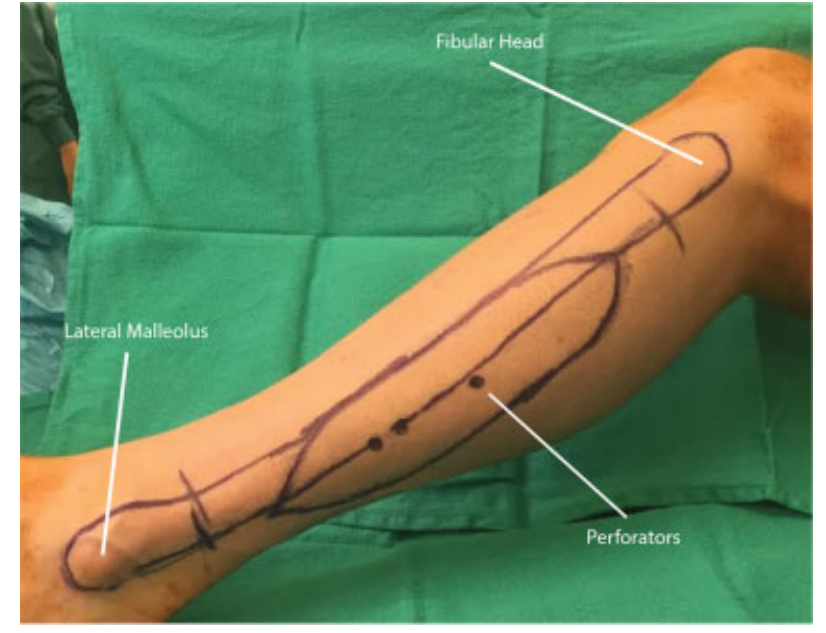

Fig. 3 Contralateral leg donor site and anatomic landmarks for flap.

Next, attention was turned to the recipient leg designated for reconstruction. Approximately $60 \mathrm{~mL}$ of bone graft was harvested from the ipsilateral proximal femoral shaft and stored for later use. The distal tibia was decorticated, the intramedullary canal was curetted, the talar dome was debrided and the defect area was irrigated with $6 \mathrm{~L}$ antibiotic impregnated pulse lavage solution. After removal of the cartilage from the talar dome, a $12 \mathrm{~mm}$ diameter by $8 \mathrm{~mm}$ deep docking whole was created in the central portion of the talar dome. The distal end of the tibial shaft was cored out creating a $12 \mathrm{~mm}$ intramedullary channel for fibular graft insertion (-Fig. 5). The proximal portion of the FVFG was beveled and impacted into the tibial shaft intramedullary canal. The distal portion was then impacted into the docking site in the talar dome. The recipient anterior tibial vessels were selected and prepared for microanastomosis. The donor fibular graft pedicle was divided and the bone appropriately aligned.

The reamed femoral bone graft was then placed around the fibular graft to further augment healing. A medial distal tibial plate, reshaped to a 90 degree L-shaped plate, was used to obtain internal stabilization for the fusion (-Fig. 6, 7). The tibial diaphyseal region was rigidly affixed to the plate with bicortical screws, while cancellous screw fixation was obtained distally into the remaining talar head, neck, and body.

Attention was then turned to the arterial anastomosis of the peroneal and anterior tibial vessels. The arterial anastomosis was performed using a double opposing disposable bulldog clamp system with 8-0 nylon sutures. The venous anastomosis was performed using a 2.5 venous Synovis coupler. The donor leg wound was partially closed, a vacuum assisted closure dressing was placed, and a posterior splint was applied. Final external stabilization was obtained on the reconstructed limb utilizing a Taylor Spatial stereotactic external fixator (-Fig. 8). This was used to obtain compression osteosynthesis through maximal compression and rigid stabilization across the tibial nonunion and ankle fusion sites, while still allowing access to, and visualization of the vascularized myocutaneous portion of the graft. 


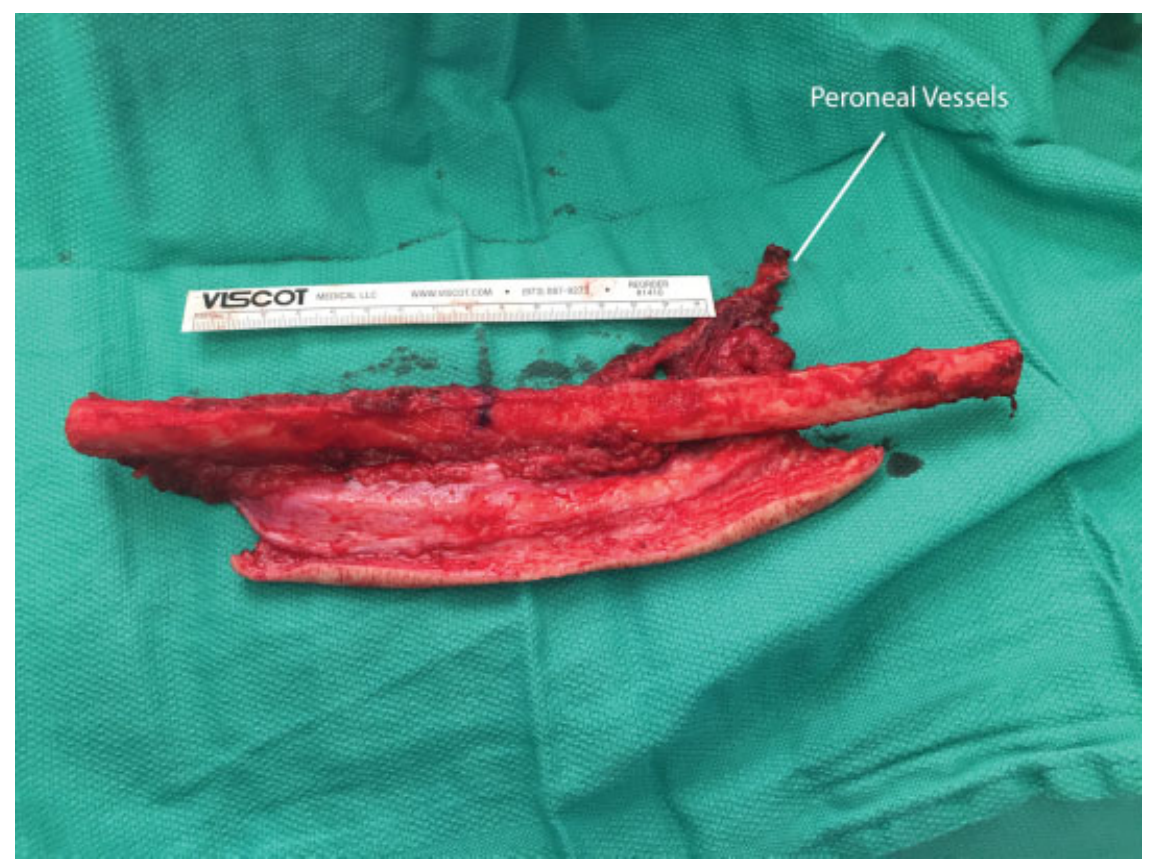

Fig. 4 Free fibula dissected with pedicle.

\section{Outcome to Date}

The patient was placed on a full-dose aspirin and heparin drip for 48 hours following surgery, and transitioned to therapeutic Lovenox for history of pulmonary embolism. A splitthickness skin graft was applied to the donor harvest site

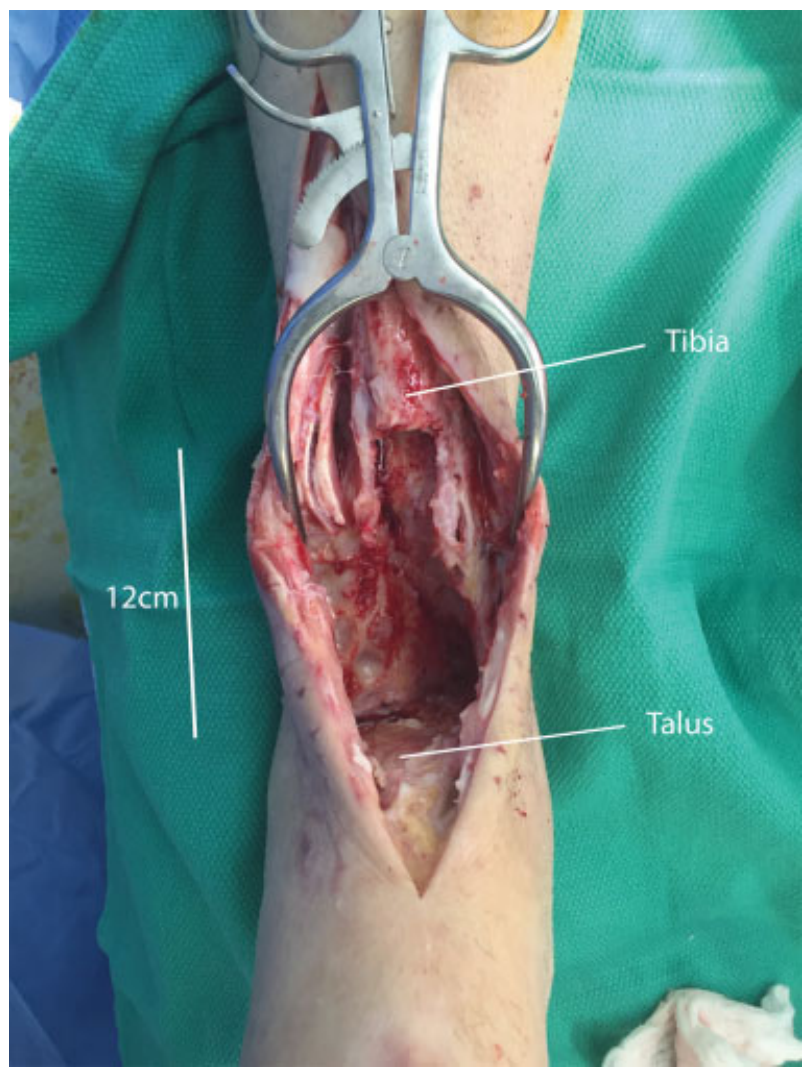

Fig. 5 Cored out distal tibia with defect visible. on postoperative day 5 . He returned to the hospital 1 month postoperatively with some wound breakdown and was treated with hyperbaric oxygen therapy to accelerate healing. The wound healed completely, and computed tomography scan confirmed successful bone fusion (-Figs. 9 and 10), at which point the external fixator was removed.

\section{Discussion}

Reconstruction of the distal tibia with ankle involvement is a challenging operation for orthopedic and plastic surgeons. Unfortunately, below-knee amputation has been the standard of care with massive tissue defects involving the ankle joint. Surgeons and patients are often faced with the difficult decision of reconstruction with limited options versus amputation. Both options have their limitations, but many

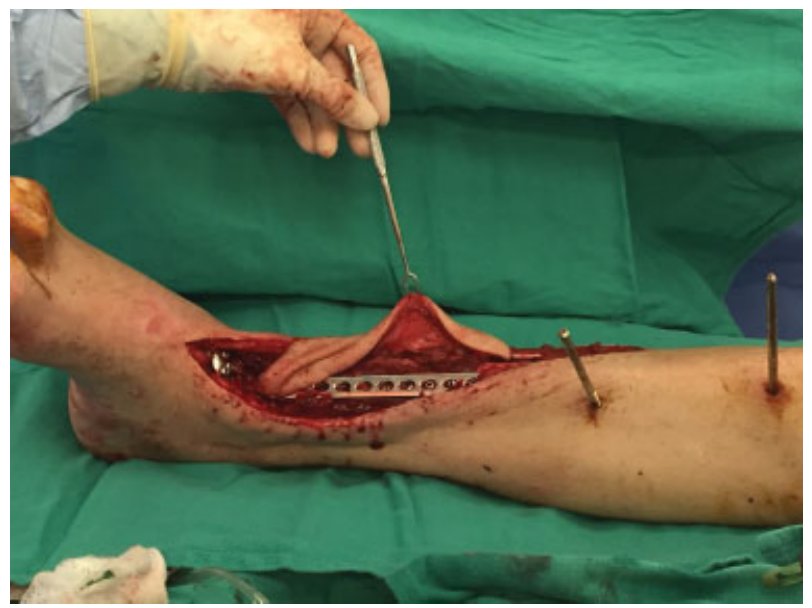

Fig. 6 Free flap in place with internal fixation plate. 


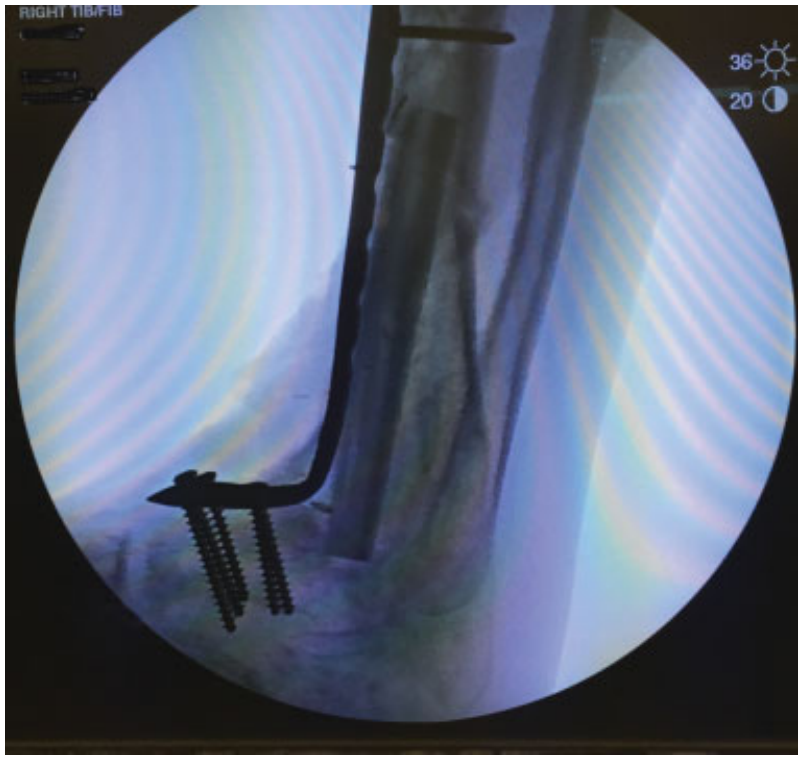

Fig. 7 Immediate postoperative X-ray revealing fibula and plate positioning.

patients prefer a limb-sparing option and have better satisfaction with a reconstruction. ${ }^{11}$ Modern prosthetic devices can restore significant function, but are not without significant drawbacks. Endoprosthetics for lower extremity defects have relatively high infections rates, and long-term use can lead to functional decline. ${ }^{12}$

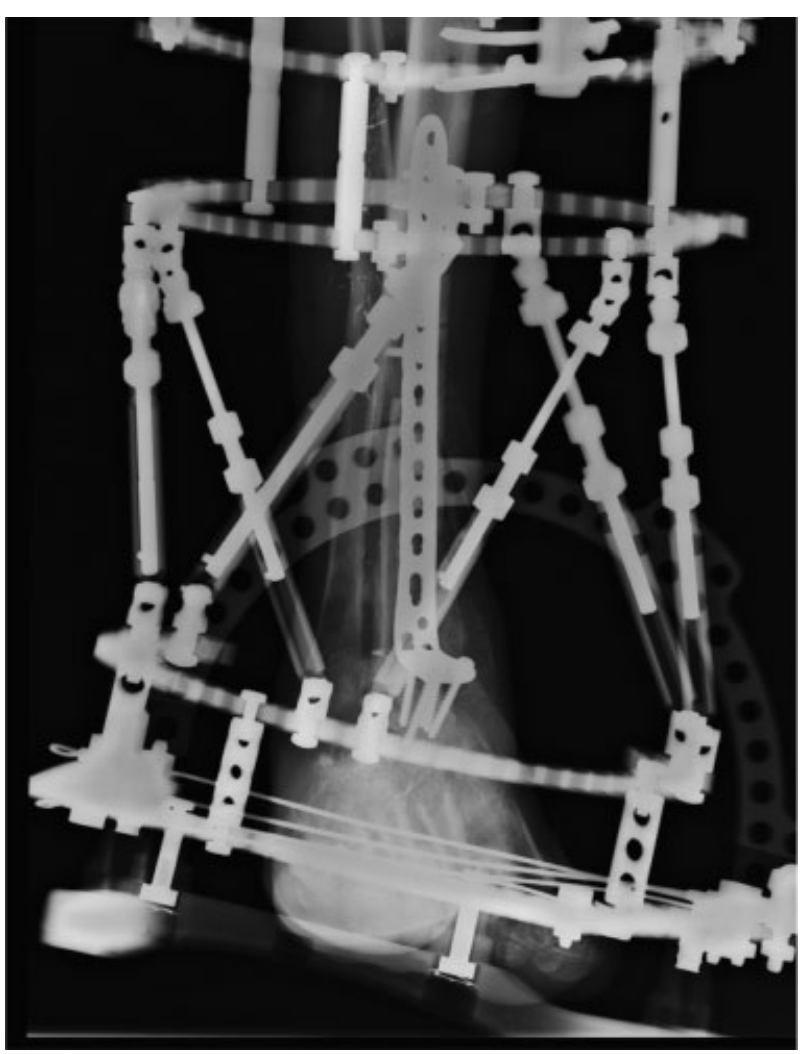

Fig. 8 X-ray post dynamic external fixator placement.

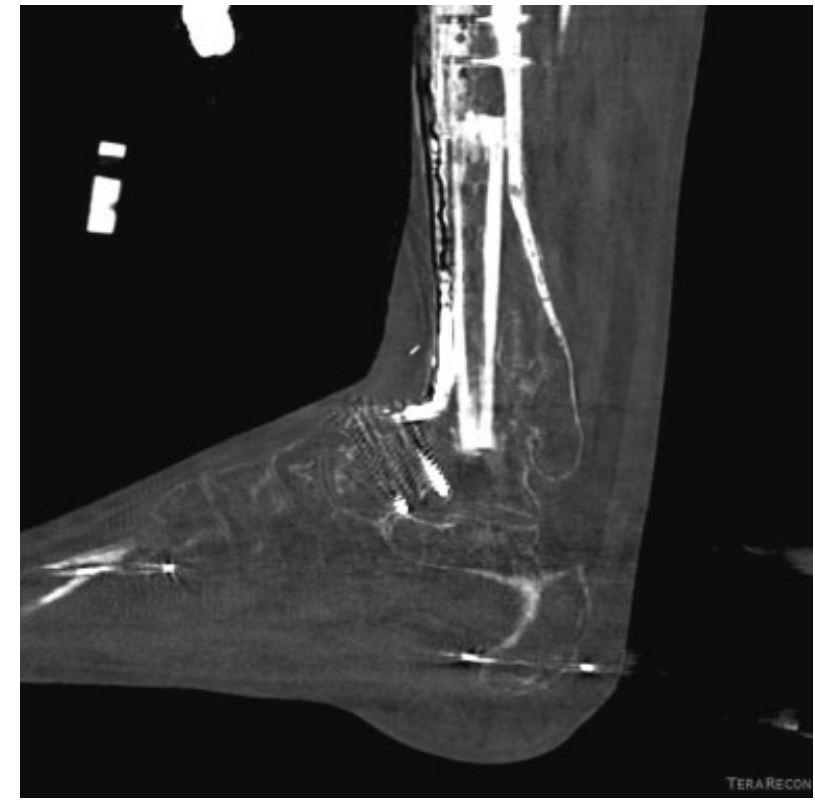

Fig. 9 Three-dimensional computed tomography reconstruction images demonstrating bony fusion.

The ankle joint is complex both anatomically and functionally. Injuries requiring large resection of the tibia often lead to structural and functional compromise. It is our belief that advancements in surgical technique can minimize these deficits. In the setting of significant bone loss, ankle arthrodesis can be remarkably difficult. Green and Roesler assessed the outcomes of pilon fractures complicated by infection. Previous attempts with extensive bone grafting to fill these defects resulted in long-term external fixation and often a pseudoarthrosis. Less than half ( 6 of 13 patients) had a

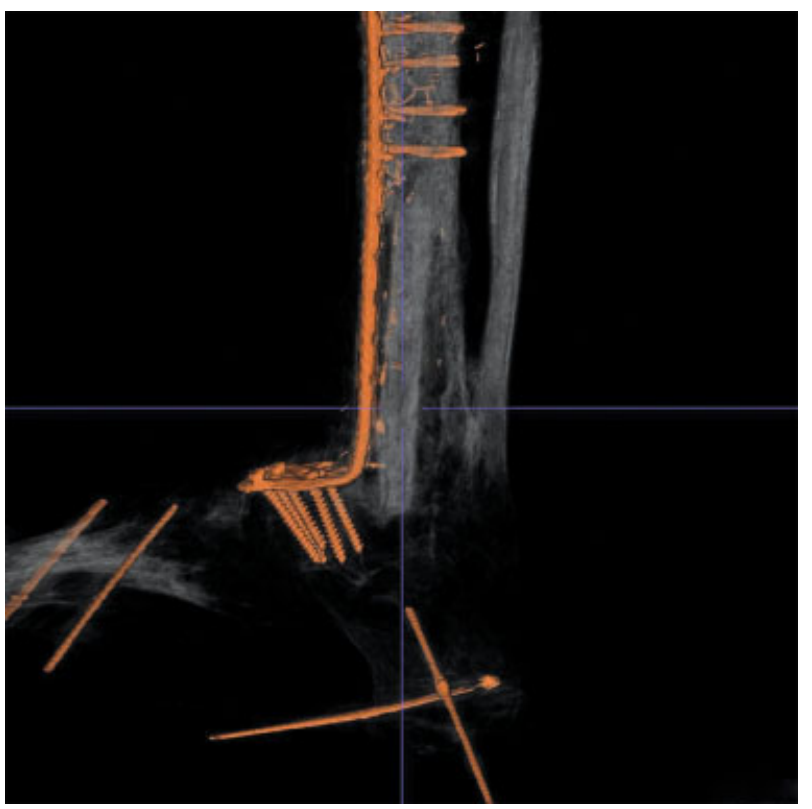

Fig. 10 Additional three-dimensional computed tomography reconstruction demonstrating bony fusion and positioning of internal hardware and fibula flap. 
successful union, and of these, all of them had stiff ankles and dystrophic skin around the distal tibia. ${ }^{13}$

Current literature shows an emerging roll for the FVFG as a reconstructive tool. We have seen great success as a structural replacement for long bones in the extremities. The various methods for harvest makes this flap a very attractive option for large, complex defects. In a recent case series, Roukis and Kang reported outcomes of tibiotalocalcaneal fusion with vascularized fibular onlay graft augmentation. They demonstrated successful augmentation of previous failed fusion using intramedullary fixation with ipsilateral vascularized fibular graft in the setting of significant bone loss and poor soft tissue quality. ${ }^{14}$ However, this technique relies on the intramedullary nail for arthrodesis and support.

In the case described, we present a patient also with a large, complex lower extremity defect which was reconstructed without the use of an intramedullary nail. To date, we have not encountered any reports of this technique for a massive bony defect involving the distal tibia and ankle joint. With this case, we present a novel and unique approach to these defects.

Previously described techniques for the FVFG have not spanned a joint in this manner. With successful union of the fibular graft to native talus, we are able to further demonstrate the versatility and reliability of this graft. It is our goal to show that these complicated ankle injuries with large defects should always be evaluated for a possible reconstruction.

While this report has been a success, we do acknowledge some limitations in this technique. Similar to all fibular transfers, length can be a limiting factor. When defects extend from the lower leg to include the ankle, length of the graft and/or length of the pedicle could become limiting. Furthermore, adequate bony material is required proximally and distally. Our patient had substantial remaining talus and was thus an adequate site for union. Traumatic injuries and malignancy can be unpredictable and establishing an adequate bony bed for distal union could be difficult or impossible.

\section{Conclusion}

Large tibial and ankle defects can be very difficult for orthopedic and plastic surgeons. We are lacking a reconstruction technique that completely restores structure and function of the joint. However, the FVFG has provided options beyond that of amputation. It is our recommendation that even joint involving defects now be evaluated for a reconstructive option. With careful patient selection, we believe the technique described can reduce the need for amputation.

\section{References}

1 Taylor GI, Miller GD, Ham FJ. The free vascularized bone graft. A clinical extension of microvascular techniques. Plast Reconstr Surg 1975;55(5):533-544

2 Chen ZW, Yan W. The study and clinical application of the osteocutaneous flap of fibula. Microsurgery 1983;4(1):11-16

3 Lee KS, Park JW. Free vascularized osteocutaneous fibular graft to the tibia. Microsurgery 1999;19(3):141-147

4 Santarelli Di Pompeo F. Tibia reconstruction. Medscape Web site. Available online at emedicine.medscape.com/article/1291028overview. Accessed January 25, 2016

5 Tomita Y, Murota K, Takahashi F, Moriyama M, Beppu M. Postoperative results of vascularized double fibula grafts for femoral pseudoarthrosis with large bony defect. Microsurgery 1994;15(5): 316-321

6 Korompilias AV, Paschos NK, Lykissas MG, Kostas-Agnantis I, Vekris MD, Beris AE. Recent updates of surgical techniques and applications of free vascularized fibular graft in extremity and trunk reconstruction. Microsurgery 2011;31(3):171-175

7 Innocenti M, Delcroix L, Manfrini M, Ceruso M, Capanna R. Vascularized proximal fibular epiphyseal transfer for distal radial reconstruction. J Bone Joint Surg Am 2004;86-A(7): 1504-1511

8 Manfrini M, Innocenti M, Ceruso M, Mercuri M. Original biological reconstruction of the hip in a 4-year-old girl. Lancet 2003; 361(9352):140-142

9 Capanna R, Bufalini C, Campanacci C. A new technique for reconstructions of large metadiaphyseal bone defects: a combine graft (allograft shell plus vascularized fibula). Orthop Traumatol 1993; 2:159-177

10 Bakri K, Stans AA, Mardini S, Moran SL. Combined massive allograft and intramedullary vascularized fibula transfer: the capanna technique for lower-limb reconstruction. Semin Plast Surg 2008;22(3):234-241

11 Davis AM, Devlin M, Griffin AM, Wunder JS, Bell RS. Functional outcome in amputation versus limb sparing of patients with lower extremity sarcoma: a matched case-control study. Arch Phys Med Rehabil 1999;80(6):615-618

12 Abudu A, Grimer RJ, Tillman RM, Carter SR. Endoprosthetic replacement of the distal tibia and ankle joint for aggressive bone tumours. Int Orthop 1999;23(5):291-294

13 Green SA, Roesler S. Salvage of the infected pilon fracture. Tech Orthop 1987;2:37-44

14 Roukis TS, Kang RB. Vascularized pedicled fibula onlay bone graft augmentation for complicated tibiotalocalcaneal arthrodesis with retrograde intramedullary nail fixation: a case series. J Foot Ankle Surg 2016; pii: S1067-2516(15)00575-X 